\title{
Primary Aldosteronism with Parathyroid Hormone Elevation: A Single-center Retrospective Study
}

\author{
Mitsuhiro Kometani ${ }^{1}$, Takashi Yoneda ${ }^{1,2}$, Daisuke Aono ${ }^{1}$, Yuko Gondoh-Noda ${ }^{1}$, \\ Takahiro Matsuoka ${ }^{1}$, Takuya Higashitani ${ }^{1}$, Shohei Yoshikura ${ }^{1}$, Kei Sawada ${ }^{1}$, \\ Yoshimichi Takeda ${ }^{1}$, Aya Fujimoto ${ }^{1}$, Shigehiro Karashima ${ }^{1}$, \\ Mikiya Usukura ${ }^{1,3}$ and Yoshiyu Takeda ${ }^{1}$
}

\begin{abstract}
:
Objective Primary aldosteronism (PA) is a major cause of secondary hypertension. The association between PA and other hormone disorders is unclear. The present study aimed to evaluate whether the parathyroid hormone $(\mathrm{PTH})$ value is associated with PA subtypes or specific treatments.

Methods We enrolled 135 patients with PA who had their PTH value measured before undergoing a specific treatment. We evaluated whether PTH value is associated with PA subtypes or with specific treatments. The present study is a single-center retrospective study (2011-2018).

Results Our study showed that, among the patients with PA, the proportion of those with PTH elevation was $>30 \%$. The PTH value was significantly correlated with both the basal plasma aldosterone concentration (PAC) and PAC after a captopril challenge test. However, the PTH value was not significantly different between the patients with unilateral and bilateral PA. We observed that the serum PTH value decreased after treatment of PA with unilateral adrenalectomy or mineralocorticoid receptor antagonists.

Conclusion Our findings suggest that the PTH value in PA patients might be associated with the autonomous production of aldosterone. However, there was no correlation between the PTH value and PA subtypes in our study. Additionally, our study showed that targeted treatment for PA may lead to a decrease in the serum PTH levels. Hence, the PTH value could potentially be used as an index for measuring the suitability for PA treatment.
\end{abstract}

Key words: primary aldosteronism, hyperparathyroidism, parathyroid hormone

(Intern Med 60: 993-998, 2021)

(DOI: 10.2169/internalmedicine.5282-20)

\section{Introduction}

Primary aldosteronism (PA) is the most frequent cause of secondary hypertension, accounting for $5-10 \%$ of patients with hypertension. In addition, patients with PA have a higher incidence of cardiovascular complications. In Japan, a recent retrospective study indicated that the prevalence of cardiovascular disease was higher among patients with PA compared to those with essential hypertension (1). Primary hyperparathyroidism (PHPT) is also one of the causes of secondary hypertension. A Scottish retrospective study revealed that patients with PHPT had a significantly increased risk of developing cardiovascular and cerebrovascular disease compared to the general population (after accounting for age and sex) (2). Therefore, excesses of aldosterone or parathyroid hormone $(\mathrm{PTH})$ are independent risk-factors that may contribute to cardiovascular complications. Furthermore, PHPT might be associated with primary aldosteronism. For example, a recent study conducted in Germany

${ }^{1}$ Division of Endocrinology and Metabolism, Department of Internal Medicine, Kanazawa University Graduate School of Medicine, Japan, ${ }^{2}$ Department of Health Promotion and Medicine of the Future, Kanazawa University, Japan and ${ }^{3}$ Department of Internal Medicine, Houju Memorial Hospital, Japan

Received for publication May 7, 2020; Accepted for publication September 28, 2020

Correspondence to Dr. Takashi Yoneda, endocrin@med.kanazawa-u.ac.jp 
found that patients with PA had higher PTH levels compared to those with essential hypertension (3), and Rossi et al. reported that the PTH value was useful for determining PA subtypes (4).

Hence, we conducted a study evaluating the association between PA and PTH elevation, and assessing the PTH level of PA patients before and after targeted treatment.

\section{Material and Methods}

\section{Patients}

Between 2011 and 2018, a total of 248 patients with PA underwent adrenal vein sampling (AVS) for subtype diagnosis at our institution. The PTH level before PA targeted treatment was measured in 135 of these patients. The diagnosis of PA was confirmed by the captopril challenge test (CCT), the furosemide plus upright test, or the saline infusion test according to the 2014 guidelines of the Japanese Society of Hypertension (JSH) (5). Further, all testing protocols adhered to the guidelines of the Japan Endocrine Society (6). The criterion for successful selective catheterization was the ratio of adrenal vein to inferior vena cava cortisol concentrations (7). The lateralization index (LI) is defined as the ipsilateral adrenal vein aldosterone to cortisol concentration ratio over the contralateral aldosterone to cortisol ratio. In pre-adrenocorticotropic hormone (ACTH) AVS, unilateral aldosterone overproduction was confirmed by a LI $>2$. In post-ACTH AVS, unilateral aldosterone overproduction was confirmed by a LI >4 (7). In total, 114 patients with PA underwent successful pre-ACTH AVS. Of these, 60 had a diagnosis of unilateral PA. In addition, 124 patients with PA underwent successful post-ACTH AVS, of whom 19 patients had a diagnosis of unilateral PA. We investigated the proportion of patients with a PTH level higher than the upper limit among PA patients, and evaluated the association between the PTH value and PA subtype. We also investigated whether specific therapies for PA had an impact on the PTH level.

We measured the PTH level using intact PTH, which includes 1-84 and 7-84 PTH fragments. Intact PTH was measured by an electrochemiluminescence immunoassay with the Cobas system (Roche Diagnostics, Tokyo, Japan) in our own hospital. The reference interval is $10.3-65.9 \mathrm{pg} / \mathrm{mL}$.

\section{Ethics and statistics}

This clinical study was approved by the Ethics Committees of Kanazawa University and all study procedures were carried out in accordance with the Declaration of Helsinki. In addition, a waiver of consent was obtained from the Ethics Committees of Kanazawa University. Statistical analyses were performed using the Excel software program 2016 (Microsoft, Seattle, USA) with the add-in software program Statcel4 (OMS, Tokyo, Japan). The Mann-Whitney $U$ test was used to compare differences between independent groups. The Wilcoxon test was used to evaluate the differ- ence between two conditions among correlated samples. Spearman rank correlation coefficients were used to determine the relationship between the two data sets. p values < 0.05 were considered to be statistically significant.

\section{Results}

\section{PTH level elevation in patients with PA}

Table presents the clinical characteristics of patients with PA. In 135 patients with PA who had their serum PTH level assessed, $50(37 \%)$ had a higher PTH level than the upper limit of the reference interval. The PTH value did not significantly correlate with the systolic blood pressure, diastolic blood pressure, aldosterone-to-renin ratio (ARR), or serum potassium level. However, there was a significant correlation between the PTH value and basal plasma aldosterone concentration (PAC) ( $\mathrm{rs}=0.22, \mathrm{p}=0.009)$ (Fig. 1). In addition, PAC after CCT was also correlated with the PTH value (rs= 0.32, p<0.001) among 130 PA patients (Fig. 1).

\section{Association between PA subtype and the PTH value}

Twenty-one of 60 (35\%) patients with unilateral PA during pre-ACTH AVS had a higher PTH level than the upper limit of the reference interval while 19 of 54 (35\%) patients with bilateral PA in pre-ACTH AVS had a higher PTH level than the upper limit of the reference interval. In contrast, when examining the results of post-ACTH AVS, eight of 19 patients $(42 \%)$ had a higher PTH level than the upper limit of the reference interval, while 37 of 105 (35\%) patients with bilateral PA in post-ACTH AVS had a higher PTH level than the upper limit of the reference interval.

Based on the results of pre-ACTH AVS, the PTH value of unilateral PA was eqivalent to bilateral PA (59 vs. 56 pg/ $\mathrm{mL}, \mathrm{p}=0.77$ ) (Table). Similarly, based on the results of postACTH AVS, the PTH value of unilateral PA was eqivalent to bilateral PA (62 vs. $58 \mathrm{pg} / \mathrm{mL}, \mathrm{p}=0.23$ ) (Table).

\section{PA with targeted treatment}

Among the 135 PA patients, 18 patients received surgical treatment, including adrenalectomy $(n=17)$ and radiofrequency ablation $(n=1)$. Of these 18 patients with surgical treatment, 7 patients had a higher PTH level than the upper limit of the reference interval before surgical treatment. While, 117 PA patients received medical treatment with a mineralocorticoid receptor (MR) antagonist. Of these 117 patients with MR antagonist, 43 patients had a higher PTH level than the upper limit of the reference interval.

In this study, of the 135 patients, 17 had PTH measured both before and after undergoing specific treatment (5 after surgical treatment, 12 after MR antagonist). The PTH levels decreased after treatment in 15 of the 17 patients, and all five surgical cases showed a decrease in PTH (Fig. 2). In addition, 9 of the 10 patients ( 5 after surgical treatment, 5 after MR antagonist) whose PTH levels were above the upper limit of normal had PTH levels that fell within the nor- 
Table. Summary of Clinical Characteristics of Patients with Primary Aldosteronism.

\begin{tabular}{lccccc}
\hline & \multirow{2}{*}{ All } & \multicolumn{2}{c}{ Pre-ACTH AVS } & \multicolumn{2}{c}{ Post-ACTH AVS } \\
& & Unilateral & Bilateral & Unilateral & Bilateral \\
\hline $\mathrm{N}(\mathrm{M} / \mathrm{F})$ & $135(69 / 66)$ & $60(31 / 29)$ & $54(27 / 27)$ & $19(12 / 7)$ & $105(52 / 53)$ \\
Age (years) & $52 \pm 11$ & $52 \pm 12$ & $52 \pm 12$ & $55 \pm 11$ & $51 \pm 11$ \\
$\mathrm{sBP}(\mathrm{mm} \mathrm{Hg})$ & $136 \pm 17$ & $134 \pm 17$ & $137 \pm 18$ & $133 \pm 18$ & $136 \pm 17$ \\
$\mathrm{dBP}(\mathrm{mm} \mathrm{Hg})$ & $85 \pm 13$ & $85 \pm 12$ & $85 \pm 14$ & $86 \pm 16$ & $84 \pm 12$ \\
$\mathrm{PAC}(\mathrm{pg} / \mathrm{mL})$ & $203 \pm 173$ & $223 \pm 233$ & $167 \pm 80$ & $349 \pm 190^{\S}$ & $169 \pm 78$ \\
$\mathrm{PRA}(\mathrm{ng} / \mathrm{mL} / \mathrm{h})$ & $0.5 \pm 0.5$ & $0.5 \pm 0.5$ & $0.6 \pm 0.2$ & $0.4 \pm 0.4$ & $0.6 \pm 0.5$ \\
$\mathrm{ARR}$ & $694 \pm 1,015$ & $851 \pm 122^{*}$ & $479 \pm 452$ & $1,664 \pm 1,526^{\S}$ & $471 \pm 360$ \\
$\mathrm{~K}(\mathrm{mEq} / \mathrm{L})$ & $3.9 \pm 0.4$ & $3.8 \pm 0.5$ & $4.0 \pm 0.3$ & $3.6 \pm 0.5$ & $3.9 \pm 0.3$ \\
$\mathrm{Ca}(\mathrm{mg} / \mathrm{dL})$ & $9.2 \pm 0.4$ & $9.2 \pm 0.4$ & $9.2 \pm 0.3$ & $9.2 \pm 0.4$ & $9.2 \pm 0.4$ \\
$\mathrm{P}(\mathrm{mg} / \mathrm{dL})$ & $3.5 \pm 0.6$ & $3.4 \pm 0.6$ & $3.5 \pm 0.5$ & $3.2 \pm 0.5$ & $3.5 \pm 0.5$ \\
$\mathrm{Cr}(\mathrm{mg} / \mathrm{dL})$ & $0.8 \pm 0.7$ & $0.8 \pm 0.9$ & $0.7 \pm 0.2$ & $0.8 \pm 0.3$ & $0.8 \pm 0.7$ \\
$\mathrm{FECa}(\%)$ & $1.0 \pm 0.6$ & $1.1 \pm 0.6$ & $1.1 \pm 0.7$ & $1.3 \pm 0.7$ & $1.0 \pm 0.6$ \\
$\mathrm{PTH}(\mathrm{pg} / \mathrm{mL})$ & $60 \pm 29$ & $59 \pm 31$ & $56 \pm 20$ & $62 \pm 18$ & $58 \pm 28$ \\
\hline
\end{tabular}

ARR: aldosterone-to-renin ratio, dBP: diastolic blood pressure, F: female, FECa: fractional excretion of calcium, M: male, N: number of patients, PAC: plasma aldosterone concentration, PRA: plasma renin activity, sBP: systolic blood pressure

Data are presented as means \pm standard deviations. ${ }^{*} \mathrm{p}<0.05$ for unilateral vs. bilateral pre-ACTH AVS. $\S_{\mathrm{p}}<0.05$ for unilateral vs. bilateral post-ACTH AVS.
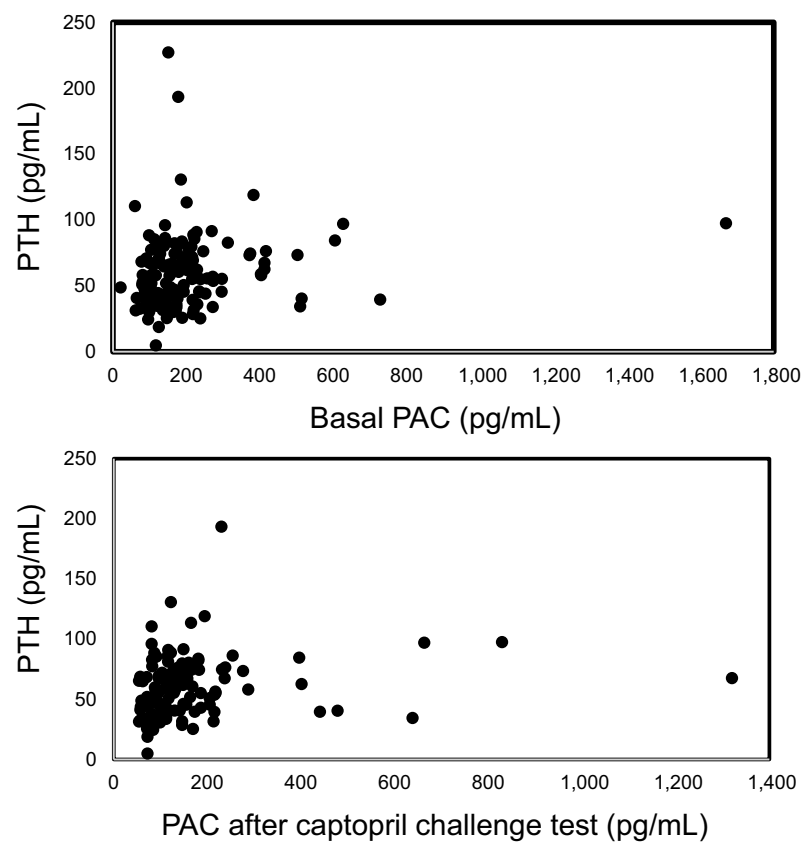

Figure 1. Association between the PTH level and the plasma aldosterone concentration. The PTH level is significantly correlated with the basal plasma aldosterone concentration (PAC) ( $r s=0.22, p=0.009$ ) (upper panel). In addition, PAC after a captopril challenge test also correlated with the PTH level (rs=0.32, $\mathbf{p}<\mathbf{0 . 0 0 1}$ ) (lower panel).

mal range after specific treatment (Fig. 2).

In addition, 16 patients had PTH and plasma renin activity (PRA) value in both before and after treatment. We found a significant negative correlation between the amount of change in PTH and PRA after specific treatment in 9 pa-
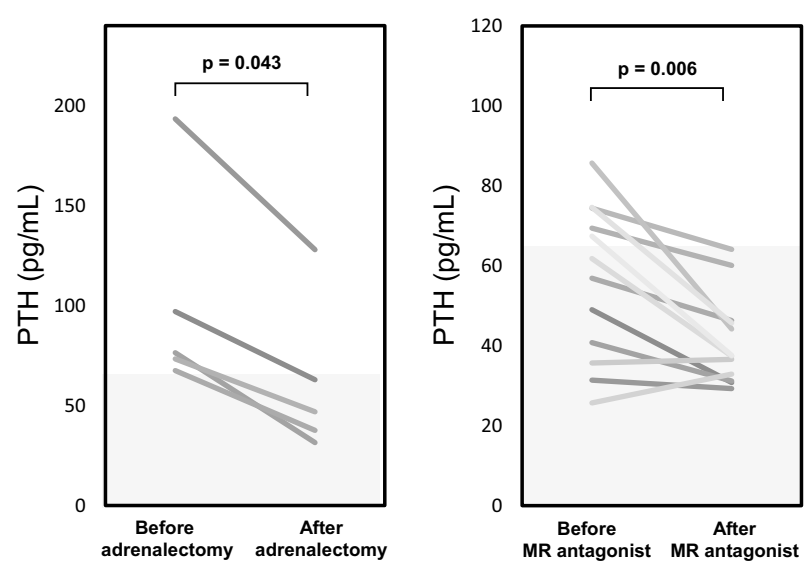

Figure 2. Changes in the PTH level before and after PA targeted treatment. In patients who underwent surgical treatment $(n=5$, left), the PTH levels were significantly lower postoperatively $(p=0.046)$. In patients treated with mineralocorticoid receptors ( $n=12$, right), the PTH levels were significantly lower after treatment $(p=0.006)$. The light gray colored area shows the reference interval of PTH.

tients with high PTH before treatment ( $\mathrm{rs}=-0.82, \mathrm{p}=0.02)$.

\section{Case description of PA with primary hyperparathy- roidism}

In addition to conducting an epidemiologic analysis among the aggregated PA patients, we also present a representative case study here. The patient was a 42-year-old Japanese woman who experienced repeated ureteroliths for 10 years. When she had a fifth ureterolith, laboratory data indicated that her serum calcium concentration was elevated 

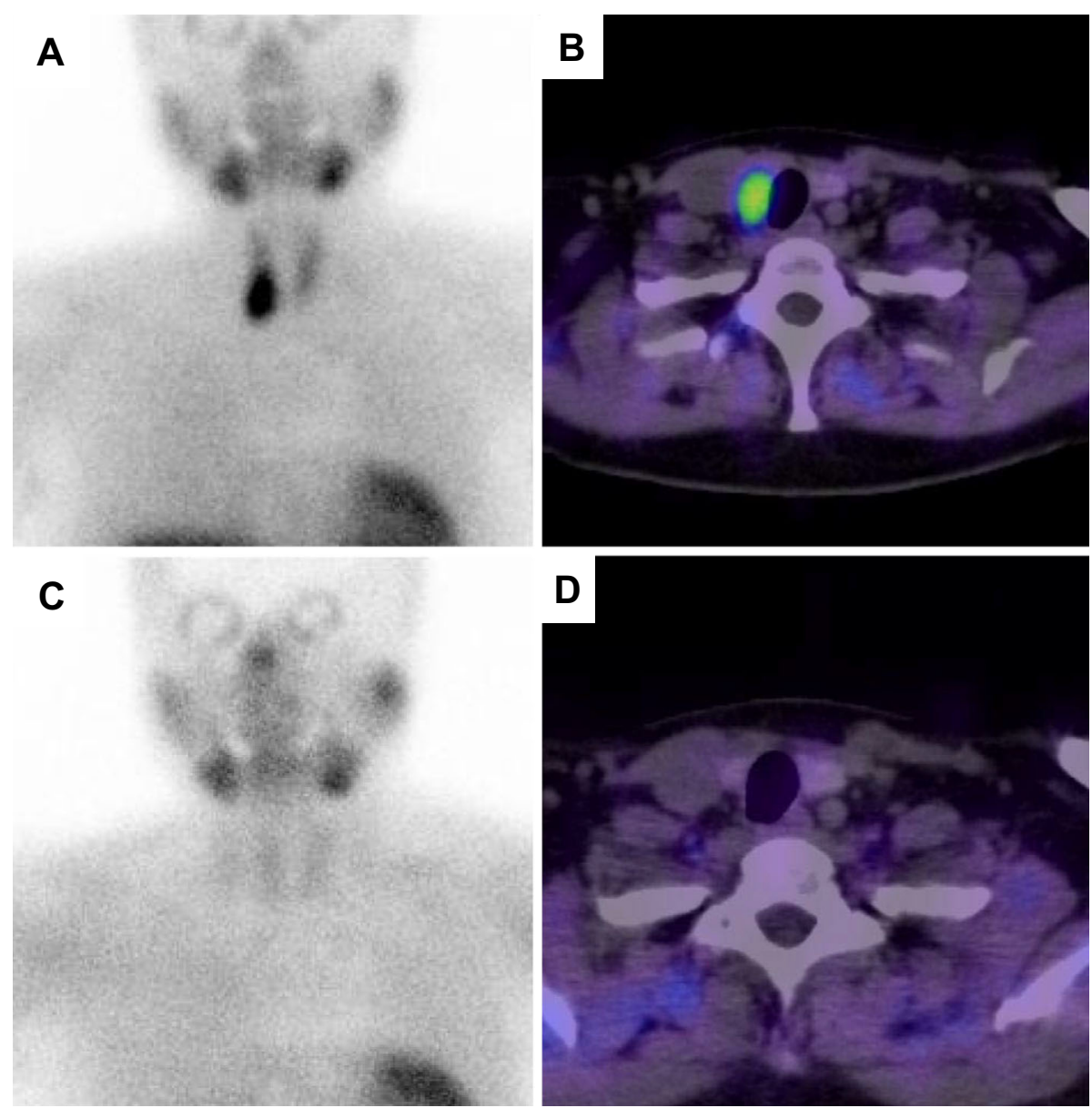

Figure 3. ${ }^{99 \mathrm{~m}} \mathrm{Tc}$-sestamibi scintigraphy of a PA patient with primary hyperparathyroidism. (A) and (B) show the preoperative state, while (C) and (D) show the postoperative state 1 year after parathyroidectomy.

to $11.1 \mathrm{mg} / \mathrm{dL}$, with a corresponding high PTH level $(200.7$ $\mathrm{pg} / \mathrm{mL}$ ). This led to hospitalization in our department. Ultrasonography showed a right parathyroid tumor $(23 \mathrm{~mm}$ diameter); scintigraphy with technetium $\left({ }^{99 \mathrm{~m}} \mathrm{Tc}\right)$ sestamibi (MIBI) demonstrated where the tumor was concentrated (Fig. 3). The patient also had hypertension; laboratory data showed that her PRA was $<0.2 \mathrm{ng} / \mathrm{mL} / \mathrm{h}$, and PAC was 136 $\mathrm{pg} / \mathrm{mL}$. CCT demonstrated that PRA was $0.3 \mathrm{mg} / \mathrm{mL} / \mathrm{h}$ and PAC was $149 \mathrm{pg} / \mathrm{mL}$ with an ARR of 497 after captopril treatment. However, CT indicated no adrenal tumor. Following surgery on the right parathyroid tumor, the PTH level initially decreased but subsequently increased again. Three months after parathyroidectomy, eplerenone treatment was started and her PTH level gradually decreased and reached the reference range after 6 months of eplerenone treatment (Fig. 4). Approximately 1 year after parathyroidectomy, MIBI scintigraphy showed no accumulation, suggesting no recurrence of hyperparathyroidism (Fig. 3).

\section{Discussion}

In our study, more than $30 \%$ of the patients with PA had a higher PTH level than the upper limit of the reference interval, which is defined as a range in which $95 \%$ of the test values are distributed. However, only one patient with PA presented with a serum calcium concentration abnormality. Our findings suggest that PTH might be influenced by PAC as well as by calcium concentrations.

The PTH reference range was established using the PTH values of 198 of 346 healthy Japanese subjects, excluding those with 25-hydroxyvitamin D concentrations of less than $15 \mathrm{pg} / \mathrm{mL}$, which suggests a relative deficiency of vitamin D (8). Several other assays were included in the report, most of which had an upper limit of $65 \mathrm{pg} / \mathrm{mL}$. The report also did not give a specific number of people. However, the reference range includes $95 \%$ of healthy people. In this study, $37 \%$ of PA patients had PTH levels above the upper limit of the reference range, an estimated percentage significantly higher than the healthy population.

Aldosterone and the PTH levels may bi-directionally influence the secretory responses for each hormone in a feedback loop. The results from an animal study suggested that excess aldosterone contributed to urinary calcium excretion, which subsequently led to secondary hyperparathyroidism (9). In a recent study of hypertensive patients, Jiang et al. found a significantly higher 24-hour urinary calcium excretion in patients with PA when compared to patients with essential hypertension; this led to lower serum calcium lev- 


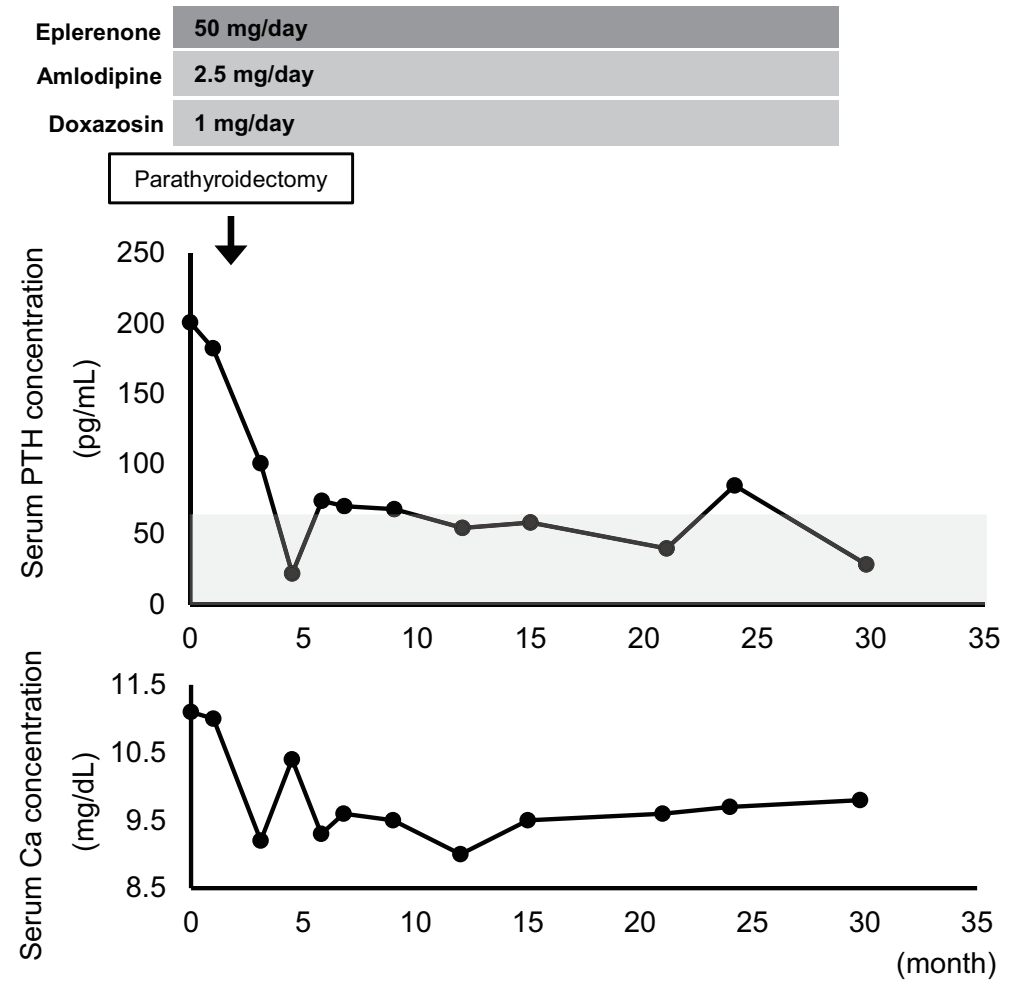

Figure 4. The clinical course of a PA patient with primary hyperparathyroidism. PTH: parathyroid hormone, Ca: calcium

els and caused an elevated PTH level among these PA patients (10). In addition, Maniero et al. reported that MR expression occurred in parathyroid-secreting cells in both PTH-secreting adenoma and the normal parathyroid gland (11). Therefore, aldosterone might act to increase PTH directly via MR. Our results indicate that the PTH value significantly correlates with both basal PAC and PAC after $\mathrm{CCT}$, thus creating an indirect relationship between these values. Conversely, Mazzocchi et al. demonstrated that PTH stimulates aldosterone secretion as a result of binding to the PTH/PTH-rP receptor in adrenal zona glomerulosa (12). Hence, research indicates that aldosterone and PTH form a positive feedback loop that might contribute to the progression of cardiovascular diseases.

The relationship between the PTH level and the PA subtype diagnosis remains to be elucidated. A previous clinical study indicated that the PTH level was useful for discriminating between subtypes of PA, which might aid physicians in deciding whether to recommend AVS to patients with PA (4). However, another clinical study indicated that the PTH level is in fact elevated in patients with PA but is irrelevant in distinguishing the subtypes of PA (10). In our study, PAC and ARR were significantly higher in unilateral PA post-ACTH AVS in comparison to bilateral PA (Table), and PAC exhibited a significant correlation with the PTH value. However, the PTH level was not significantly different between unilateral and bilateral PA cases. Further studies are needed to determine how the PTH level in patients with PA is associated with the pathophysiology of PA.

Specific treatment for PA might lead to decreased PTH levels in patients with PA (3). For example, the Graz Endocrine Causes of Hypertension (GECOH) study showed that PA treatment suppressed the PTH levels. In the GECOH study, the PTH levels in patients with PA treated with MR antagonists or adrenalectomy were similar to those in patients with essential hypertension. Another study revealed that PA treatment, such as adrenalectomy or medical therapy, reduced urinary calcium excretion and increased the serum ionized calcium concentration; this led to the normalization of the PTH levels (10). Even in this study, the PTH level decreased with respect to the reference interval after adrenalectomy or MR antagonist treatment.

A PAC decrease is one of the targeted outcomes of adrenalectomy. However, no treatment index for administering medication has yet been established. Currently, the blood pressure or serum potassium measures are the only indices typically utilized. We previously reported that longterm MR blocker treatment results in remission in some patients with PA (13). However, the mechanism through which this occurs as well as the patients' backgrounds are unknown. A recent clinical cohort study indicated that patients with PA whose PRA remained suppressed while on MR antagonists had a significantly higher risk for both cardiovascular events and death than patients with essential hypertension. This demonstrated that an unsuppressed PRA level is an important target for the treatment of patients with PA (14). In the case profile presented in the present study, the patient's PTH level increased to more than the upper limit of the reference interval. Before her hospital visit at 24 months, the eplerenone treatment dose had been insufficient. 
Moreover, the patient was not regularly taking her medication. Hence, insufficient medication may have raised her PTH level and adequate medication resulted in her PTH level decreasing to the reference level. Furthermore, in this study, the amount of change in PTH and PRA was significantly inversely correlated with specific treatments, although only in cases where PTH was above the upper limit of the reference value. Therefore, the PTH level after targeted PA treatment may be one of factors reflecting treatment efficacy, similar to PRA. Furthermore, the presence of PA should be suspected in cases in which a high PTH level is prolonged regardless of parathyroidectomy.

In summary, our study showed that there was no correlation between the PTH level and PA subtype. However, the basal PAC levels as well as levels after captopril treatment were significantly correlated with the PTH value. Our results suggest that the PTH value in PA patients may be associated with the autonomous production of aldosterone. In addition, we described a PA case profile, in which targeted treatment for PA may have led to decreased PTH levels. A decrease in PTH therefore has the potential to become an index for measuring the effectiveness of PA treatment.

The authors state that they have no Conflict of Interest (COI).

\section{References}

1. Ohno Y, Sone M, Inagaki N, et al. Prevalence of cardiovascular disease and its risk factors in primary aldosteronism: a multicenter study in Japan. Hypertension 71: 530-537, 2018.

2. Yu N, Donnan PT, Flynn RW, et al. Increased mortality and morbidity in mild primary hyperparathyroid patients. The Parathyroid Epidemiology and Audit Research Study (PEARS). Clin Endocrinol (Oxf) 73: 30-34, 2010.

3. Pilz S, Kienreich K, Drechsler C, et al. Hyperparathyroidism in patients with primary aldosteronism: cross-sectional and interventional data from the GECOH study. J Clin Endocrinol Metab 97:
E75-E79, 2012.

4. Rossi GP, Ragazzo F, Seccia TM, et al. Hyperparathyroidism can be useful in the identification of primary aldosteronism due to aldosterone-producing adenoma. Hypertension 60: 431-436, 2012.

5. Shimamoto K, Ando K, Fujita T, et al. Japanese Society of Hypertension Committee for Guidelines for the Management of Hypertension. The Japanese Society of Hypertension Guidelines for the Management of Hypertension (JSH 2014). Hypertens Res 37: 253390, 2014.

6. Nishikawa T, Omura M, Satoh F, et al. Guidelines for the diagnosis and treatment of primary aldosteronism-the Japan Endocrine Society 2009. Endocr J 58: 711-721, 2011.

7. Kometani M, Yoneda T, Aono D, et al. Impact of aldosteroneproducing cell clusters on diagnostic discrepancies in primary aldosteronism. Oncotarget 9: 26007-26018, 2018.

8. Inomata $\mathrm{K}$, Yamashita H, Yamamoto A, et al. [Involvement of 25hydroxyvitamin D in setting the intact PTH reference range]. Osteoporosis Japan 12: 449-456, 2004 (in Japanese).

9. Chhokar VS, Sun Y, Bhattacharya SK, et al. Hyperparathyroidism and the calcium paradox of aldosteronism. Circulation 111: 871878, 2005.

10. Jiang $\mathrm{Y}$, Zhang $\mathrm{C}$, Ye L, et al. Factors affecting parathyroid hormone levels in different types of primary aldosteronism. Clin Endocrinol (Oxf) 85: 267-274, 2016.

11. Maniero C, Fassina A, Guzzardo V, et al. Primary hyperparathyroidism with concurrent primary aldosteronism. Hypertension $\mathbf{5 8}$ : 341-346, 2011.

12. Mazzocchi G, Aragona F, Malendowicz LK, Nussdorfer GG. PTH and PTH-related peptide enhance steroid secretion from human adrenocortical cells. Am J Physiol Endocrinol Metab 280: E209E213, 2001.

13. Yoneda $\mathrm{T}$, Demura $\mathrm{M}$, Takata $\mathrm{H}$, et al. Unilateral primary aldosteronism with spontaneous remission after long-term spironolactone therapy. J Clin Endocrinol Metab 97: 1109-1113, 2012.

14. Hundemer GL, Curhan GC, Yozamp N, Wang M, Vaidya A. Cardiometabolic outcomes and mortality in medically treated primary aldosteronism: a retrospective cohort study. Lancet Diabetes Endocrinol 6: 51-59, 2018.

The Internal Medicine is an Open Access journal distributed under the Creative Commons Attribution-NonCommercial-NoDerivatives 4.0 International License. To view the details of this license, please visit (https://creativecommons.org/licenses/ by-nc-nd/4.0/).

(C) 2021 The Japanese Society of Internal Medicine

Intern Med 60: 993-998, 2021 